\title{
ANALYSIS OF PROACTIVE AND REACTIVE MANET ROUTING PROTOCOLS UNDER SELECTED TCP VARIANTS
}

\author{
Iffat Syad $^{1}$, Sehrish Abrejo ${ }^{2}$ and Asma Ansari ${ }^{3}$ \\ ${ }^{1}$ Department of Computer Science, Model College for Girls, Islamabad \\ iffatsheerazi@hotmail.com \\ ${ }^{2}$ Department of Computer Science, Isra University, Hyderabad \\ Sehrish.abrejodisra.edu.pk \\ ${ }^{3}$ Department of Computer Science, Isra University, Hyderabad \\ asma.ansarieisra.edu.pk
}

\begin{abstract}
A Mobile Ad hoc Network (MANET), with its inherent dynamic and flexible architecture, demonstrates attractive potential for military applications. It is able to overcome traditional communications limitations through its automatic relaying and self-healing/forming features. MANET nodes perform the routing functions themselves. Due to the limited wireless transmission range, the routing generally consists of multiple hops. Therefore, the nodes depend on one another to forward packets to the destinations. In a Mobile Ad Hoc Network (MANET), temporary link failures and route changes happen frequently. With the assumption that all packet losses are due to congestion, Transport Control Protocol (TCP) performs poorly in such an environment. One problem of TCP in such environments is its inability to distinguish losses induced by the lossy wireless channel from the ones due to network congestion. Many TCP variants have been developed for the improved performance of TCP in MANET. In this research, through simulations that were carried out by using Network Simulator-2 (NS-2), the selected MANET Routing protocols i.e. DSR and DSDV were analyzed in accordance with their finest performance of packets delivery rate, average end-to-end delay, and packet dropping, under TCP Vegas and TCP Newreno with mobility consideration. The simulation results indicate that DSDV has a better throughput performance but higher average end-to-end delay and packet drop ratio as compared to DSR
\end{abstract}

\section{KEYWORDS}

Mobile Ad hoc Networks, TCP Variants, Routing Protocols

\section{INTRODUCTION}

Mobile Ad hoc Network (MANET) is a collection of self-configuring mobile node without any infrastructure network [1]. In this network, mobile nodes communicate with each other without any base station. In the absence of base station, mobile nodes use multi-hop path from source to destination, in which nodes can act as routers to forward data. Each of the nodes contains a wireless interface. These nodes communicate over either radio or infrared. If a mobile node is within the range of other mobile, then a route is established as show in Figure 1.

DOI : $10.5121 /$ ijasuc.2013.4402 
Ad hoc networking can be deployed anywhere where there is no communication or the existing infrastructure is inconvenient and expensive to use. Typical applications where MANET can be used are Military Battlefield, Personal Area Network (PAN), Commercial Sector, Emergency search-and-rescue operations etc. It is featured by dynamic topology (infrastructure-less), multihop communication, limited resources (bandwidth, CPU, battery, etc.) and limited security [2]. Due to its dynamic nature, a mobile node can leave or join the network at any time, which can cause the route to be discovered again. To find and maintain an optimal route, many routing algorithms have been proposed. These routing algorithms are categorized as Proactive and Reactive. Proactive routing algorithms are those which maintain routing tables and keep track of changes in the network, so the route is available at the time of data transmission. On the other hand, reactive routing algorithms are used to establish a route on-demand, i.e. when one node wants to send data to other node within the network.

In traditional wired networks, TCP/IP protocol was designed to provide reliable end-to-end communication between nodes. But in case of ad hoc networks, the packet loss is due to congestion in the network and due to frequent link failures. TCP performs poor in such environments. So many variants have been proposed to improve its performance. This research is carried out to investigate the performance of two MANET routing protocols; namely DSDV and DSR under TCP Vegas and TCP Newreno on the basis of Packet Delivery Ratio, End-to-end Delay and Packet Drop.

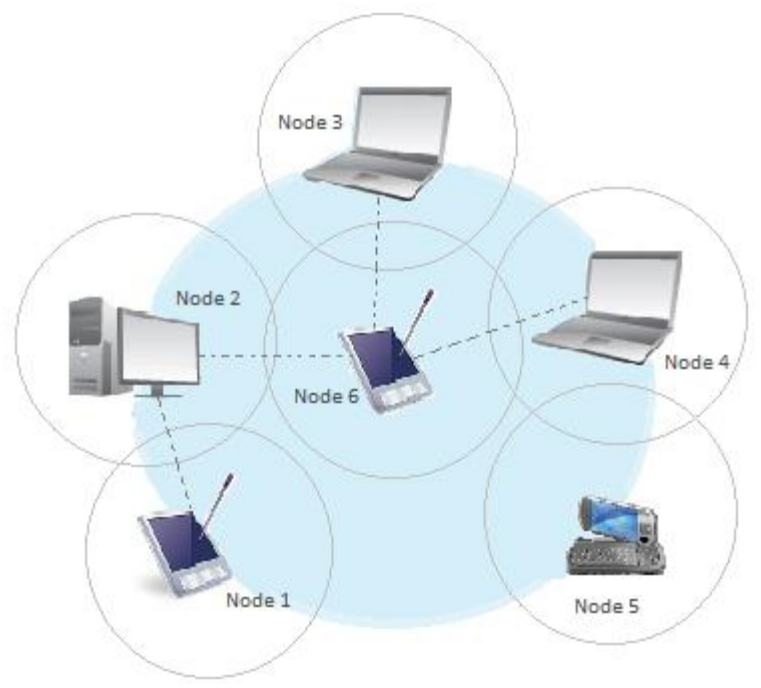

Figure1. Mobile Ad hoc Network with six wireless nodes

The remainder of the paper is organized as follows: section 2 briefly reviews the two routing protocols, DSR and DSDV; section 3 covers simulation parameters used for simulations; section 4 is used to describe the simulation performance of two routing protocols; section 5 presents discussion and section 6 concludes this paper.

\section{ROUTING IN MANETS}

The routing is the act of moving information (packets) across a network from a source to a destination. 
International Journal of Ad hoc, Sensor \& Ubiquitous Computing (IJASUC) Vol.4, No.4, August 2013

The routing infrastructure needs to be established in a distributed, self-organized way due to node mobility. The MANET routing protocols can be divided into three categories [3]. Such protocols must deal with the typical limitations of these networks, which include high power consumption, low bandwidth, and high error rates. These routing protocols derived from distance vector or link state [4] routing algorithms may be generally categories as:

- Proactive protocols or table driven protocols

- Reactive protocols or on-demand protocols

- Hybrid Routing protocols

\subsection{Proactive Routing Protocols}

Proactive routing protocols are distance-vector [5] and link-state based. These routing protocols attempt to maintain consistent, up-to-date routing information from each node to every other node in the network. These protocols require each node to maintain one or more tables to store routing information, and they respond to changes in network topology by propagating updates throughout the network in order to maintain a consistent network view. The areas in which they differ are the number of necessary routing-related tables and the methods by which changes in network structure are broadcast.

\section{Advantages}

In proactive routing protocols, any time when a route is needed, a route is already available in the table. Proactive or table driven protocols can reduce the average delay per packet.

\section{Disadvantages}

However, purely proactive schemes are not appropriate for reconfigurable wireless ad hoc network environment, as they continuously use a large portion of the network capacity to keep the routing information current. Since the node movement may be quite fast and the topology changes may be more frequent than the route requests, most of this routing information may never be used. This results in a further waste of the network capacity.

The following section describes the DSDV routing protocol in detail because as DSDV is used in the simulations.

\subsubsection{Destination-sequenced Distance-vector Routing Protocol (DSDV)}

Distance-Sequenced Distance-Vector (DSDV) [6] is a table driven routing protocol for mobile ad-hoc networks which is based on the Bellman-Ford algorithm. Each node in the network maintains a routing table in which is listed every destination and the number of hops required to reach that destination. In addition each entry in the routing table also includes a sequence number which is given by the destination node. When a node is starting to send information it will choose on-first-basis the route to destination with the most recent sequence number. It is more likely that the most recently discovered route is still active compared to older ones. If there is more than one route to the same destination with the same sequence number, then the path with the lowest hop count is chosen. 
Each node in the network periodically transmits updates to other nodes about the current routing table. The update packets can include the whole routing table of the node or just some routing information. The packets are broadcast to nearby neighbors and upon receiving them each node updates its own routing table. In the update procedure each node increments the number of hops, updates the sequence number and possibly updates the address of the next router for each destination. After the update procedure each node broadcasts its updated routing table forward. The update packets can contain the addresses either as MAC addresses or as network addresses. In between timed broadcasts of routing tables there are two different ways to send routing information to other nodes. First is a full-dump where a node sends all of its routing information and the second is an incremental where the node only sends information which has changed since the last full-dump. Routing information is sent in Network Protocol Data Units (NPDU). An incremental update should be small enough to fit into one NPDU. Full-dump probably takes more than a single NPDU depending on the size of the ad-hoc network. When there is only small amounts of movement in the ad-hoc network full-dumps should be quite infrequent, incremental updates should be enough to keep the routing tables in order. A full-dump should be used instead of an incremental update if the size of the incremental update approaches the size of the NPDU. This might happen when there is much movement in a large ad-hoc network and therefore requires many route updates. When a node receives either an incremental or a full dump routing table update it will compare the information to its current routing table. If it contains new route information it will store that route and increment the hop count by one. Nodes always use the most recent information for every route. After the node has processed all of the route updates in the NPDU it will send the changed routing table updates to its neighbors.

When nodes move across the ad-hoc network they can cause broken links in the routing tables. When a node notices that a neighbor node has moved, it will change all of its routing tables hop counts to infinity where the next router was that particular node and also update the sequence number of those routes to an odd number. It will then inform its neighbors that it no longer has a route to that node by sending a route update where the sequence number is an odd number and the hop count is infinite. An odd sequence number is used to reflect that the path is infinite e.g. no longer reachable. Even sequence numbers are used to inform of a working route.

\subsection{Reactive Routing Protocols}

A different approach from table-driven routing is reactive or on-demand routing [7]. This type of routing creates routes only when desired by the source node. When a node requires a route to a destination, it initiates a route discovery process within the network. This process is completed once a route is found or all possible route permutations have been examined. Once a route has been established, it is maintained by a route maintenance procedure until either the destination becomes inaccessible along every path from the source or until the route is no longer desired.

The following sections discuss some of the existing on-demand ad hoc routing protocols.

\subsubsection{Dynamic Source Routing}

DSR is a simple and efficient routing protocol designed specifically for use in multi-hop wireless ad hoc networks of mobile nodes. DSR allows the network to be completely self-organizing and self-configuring, without the need for any existing network infrastructure or administration. The protocol is composed of the two main mechanisms 
- Route Discovery

- Route Maintenance

Which work together to allow nodes to discover and maintain routes to arbitrary destinations in the ad hoc network. All aspects of the protocol operate entirely on demand, allowing the routing packet overhead of DSR to scale automatically to only what is needed to react to changes in the routes currently in use. The protocol allows multiple routes to any destination and allows each sender to select and control the routes used in routing its packets, for example, for use in load balancing or for increased robustness. Other advantages of the DSR protocol include easily guaranteed loopfree routing, operation in networks containing unidirectional links, use of only "soft state" in routing, and very rapid recovery when routes in the network change. The DSR protocol is designed mainly for mobile ad hoc networks of up to about two hundred nodes and is designed to work well even with very high rates of mobility.

\section{SIMULATION SETUP}

The main objective on this research is to analyze the performance of selected two MANET routing protocols destination sequenced distance vector routing protocol (DSDV) and Dynamic Source Routing DSR under two different TCP variants these are TCP-Newreno and TCP-Vegas. The analysis work is carried out by the means of a NS2-simulator. Table 1 summarizes various parameters use to setup simulation environment.

Table 1. Simulation Parameters

\begin{tabular}{||l|c|l|c||}
\hline \multicolumn{1}{|c|}{ Variables } & Value & \multicolumn{1}{c|}{ Variables } & Value \\
\hline Transmission range & $250 \mathrm{~m}$ & Traffic type & TCP \\
\hline Simulation time & $900 \mathrm{~s}$ & Packet rate & 4 packets $/ \mathrm{sec}$ \\
\hline Topology size & $1000 \mathrm{~m} \times 800 \mathrm{~m}$ & Packet size & $512 \mathrm{bytes}$ \\
\hline Total nodes & 50 & Maximum Speed & $20 \mathrm{~m} / \mathrm{s}$ \\
\hline Mobility model & Random Waypoint & Number of sources & 10 \\
\hline Pause time & $10 \mathrm{~s}, 250 \mathrm{~s}, 700 \mathrm{~s}$ & NS-2 Version & NS-2.34 \\
\hline
\end{tabular}

\section{SiMULATION RESULTS}

This section describes the results achieved from the simulations. To analyze the affect of simulations, 10 mobility scenario files were generated for pause-time 10s, 250s and 700s. Furthermore, the traffic load is fixed to 10 sources, generating 4 packets per second. The results generated by simulator in the trace files were analyzed with the help of AWK script. There are three performance metrics that are measured in these simulations, namely, packet delivery fraction, average end-to-end delay and Total Packet Dropped.

\subsection{Packet Delivery Ratio (PDF)}

The PDF is defined as the number of received data divided by the number of packets generated. For good performance and increased throughput, higher PDF shows the effectiveness of any protocol in MANET. In these study two protocols namely DSR and DSDV are analyzed under TCP Vegas and TCP Newreno. On analyzing the graph of Packet Delivery ratio (Figure 2, 3), 
results shown that DSDV have good performance and increased throughput, in most of the cases as compared to DSR.

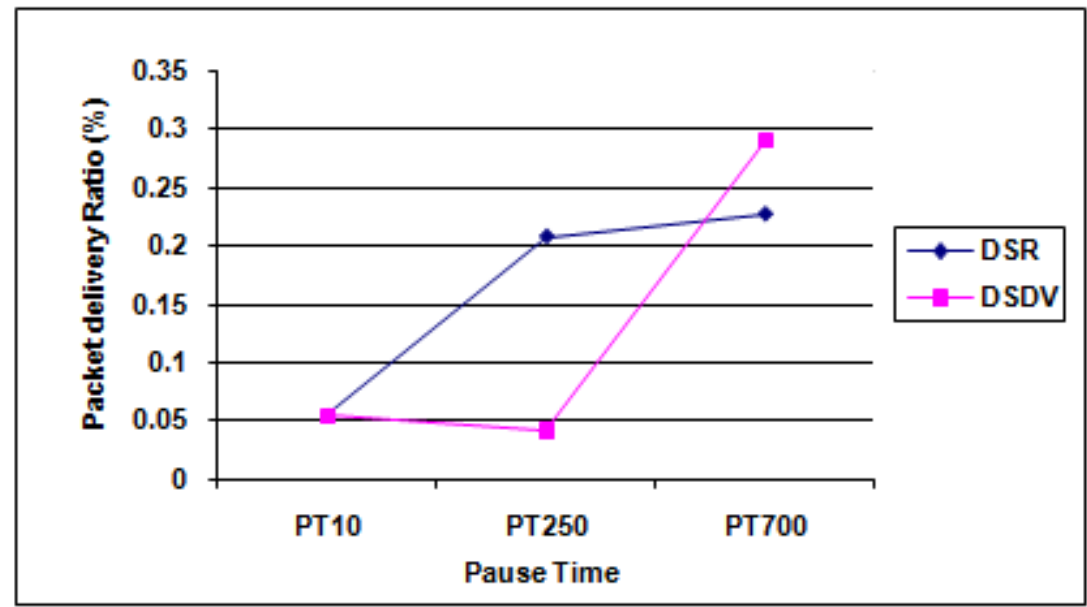

Figure 2. PDF in TCP Newreno

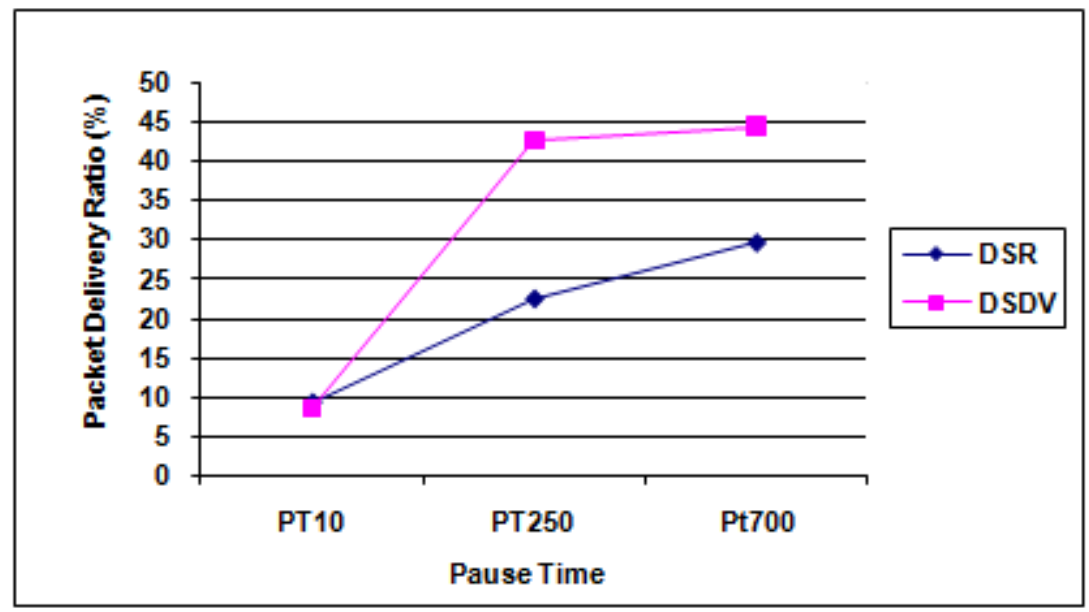

Figure 3. PDF in TCP Vegas

\subsection{Average End-to-End Delay}

The End-to-End Delay is defined as the time a data packet takes to travel from source to the destination. Average End-to-End Delay is the delay perceived by all the packets including route acquisition delay. Figure 4 and Figure 5 show, the average End-to end delay for two selected MANET routing protocols namely DSR, and DSDV. It shows variations in the graph, DSDV has highest End-to-End delay at pause time 10, because DSDV protocol keeps packets in queues indefinitely until they are delivered to the next hop or the destination node. Therefore, it delivers the older packets rather than the newer ones, and hence there is an increase in average end- toend delay for DSDV protocol, and having lowest End-to-End delay at pause time 250 and in TCP Newreno highest End-to-delay was at pause time 250. 
International Journal of Ad hoc, Sensor \& Ubiquitous Computing (IJASUC) Vol.4, No.4, August 2013

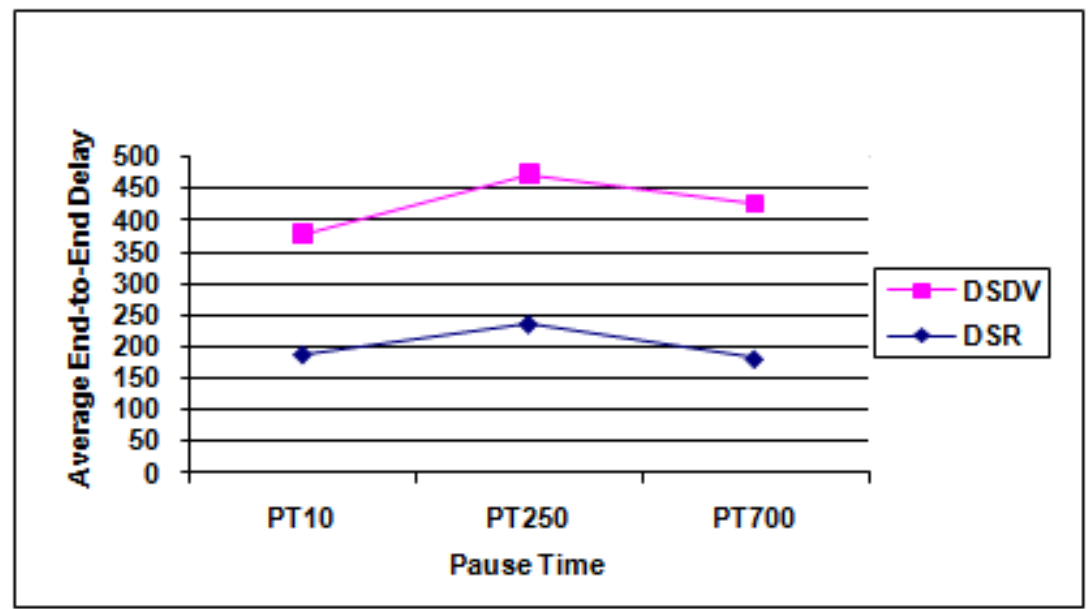

Figure 4. Average End-to-End Delay in TCP Newreno

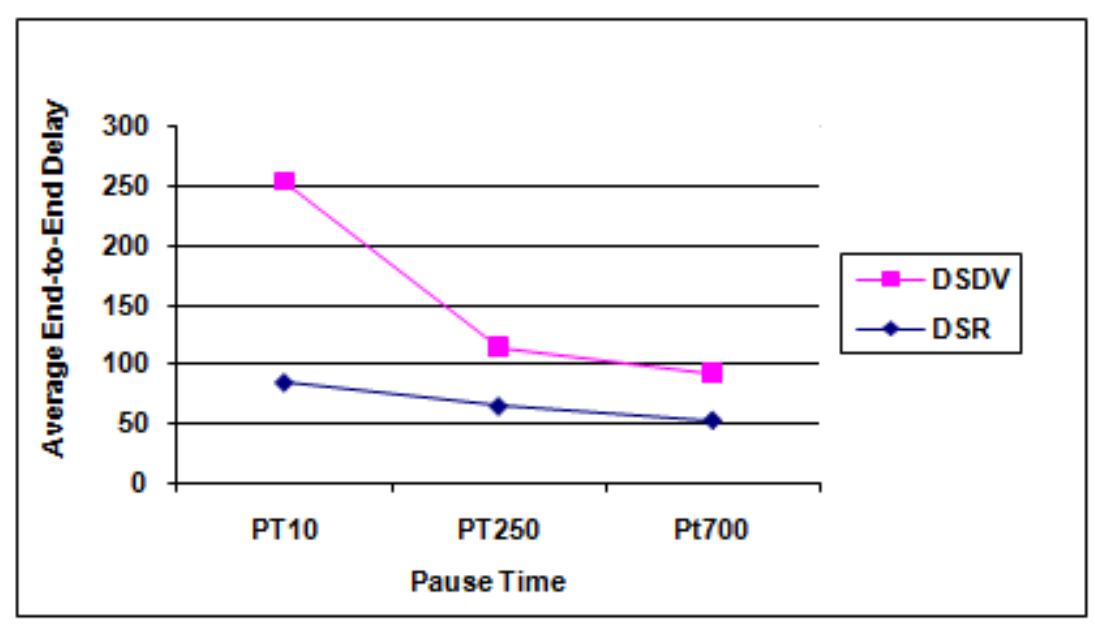

Figure 5. Average End-to-End Delay in TCP Vegas

\subsection{Total Packet Dropped}

Since wireless is a loose environment and especially when there is MANET, connection formation and deformation is usually present. So packet dropping refers to the loss of packet due to no connection availability or nodes are not in the range of each other. As mentioned previously that TCP Vegas is a TCP congestion control, or network congestion avoidance, algorithm that emphasizes packet delay, rather than packet loss, as a signal to help determine the rate at which to send packets. As shown in Figure 6 that DSDV has highest packet dropped at pause time 250 because of high mobility as compared to DSR which has lowest packet drop. Same is the case in TCP Vegas as shown in Figure 7. 
International Journal of Ad hoc, Sensor \& Ubiquitous Computing (IJASUC) Vol.4, No.4, August 2013

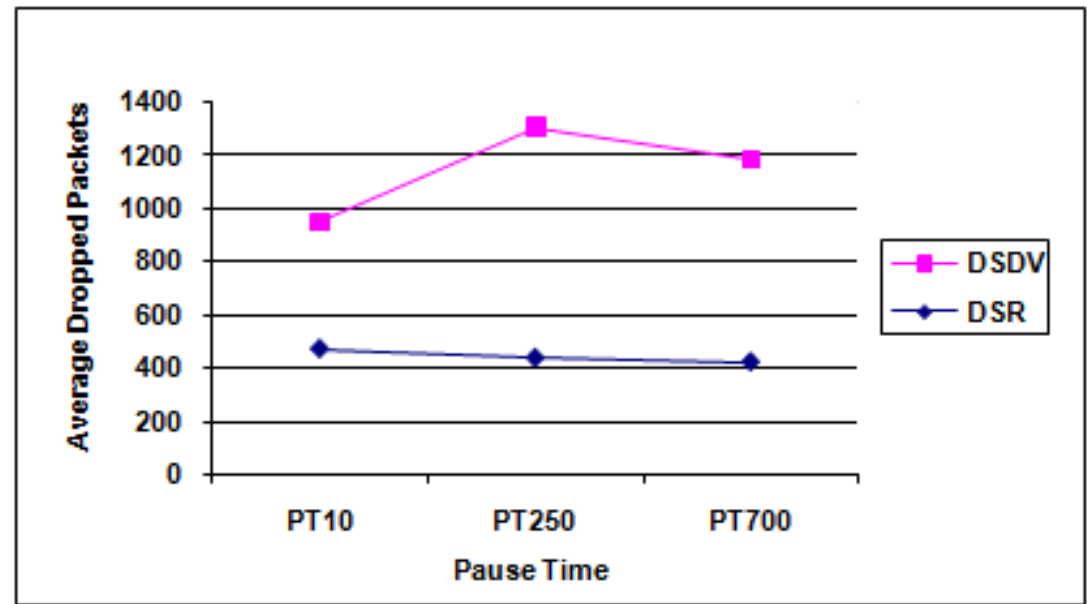

Figure 6. Total Packets Dropped in TCP Newreno

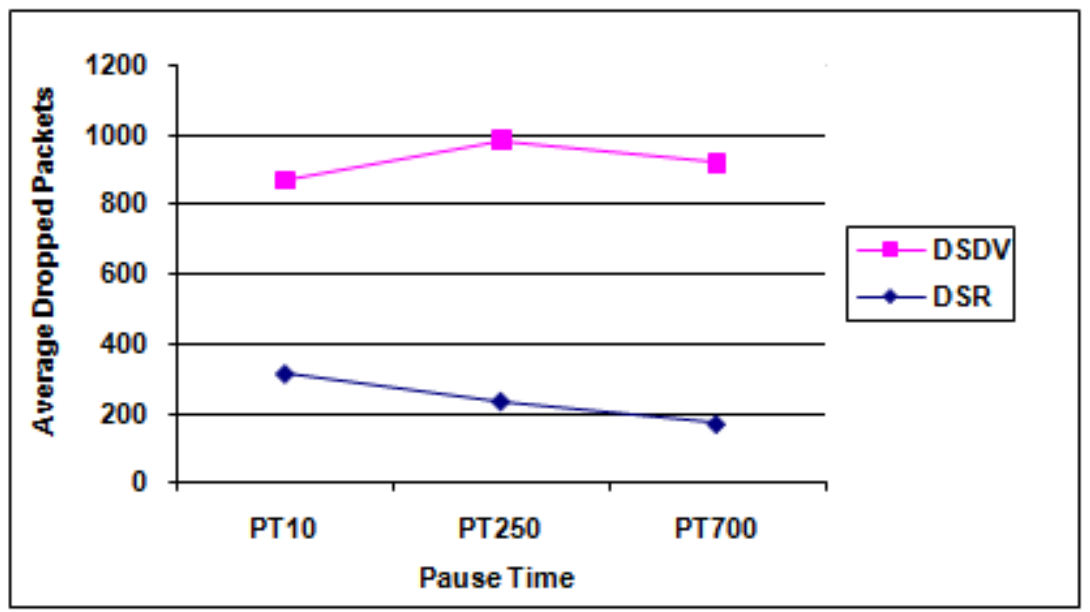

Figure 7. Total Packets Dropped in TCP Vegas

\section{DISCUSSIONS}

Following conclusions were obtained after rigorous simulations through NS-2 for analysis of two MANET Routing Protocols under two TCP variants:

Since in this study, the major concern is related with TCP type of traffic, so the reception of TCP packets is involved in the results. On analyzing the graph of Packet delivery rate (Figure 2), results shows that DSDV has the highest packet delivery ratio and because of the highest PDF its performance and throughput is better than DSR in both chosen TCP variants, the reason that DSDV has the highest PDF is that in DSDV, efficacious route cache management is done via a cache entry timeout that ensures that only active routes are maintained in the route cache. This prevents the problem of a stale route entry cache. Also the use of sequence numbers prevents the formation of routing loops; other reason is that DSDV is distance vector routing protocol, searches for route are done only when needed. In critical analysis, Vegas are giving linear delivery rate. The other important point to be noted from (Figure 2) is the convergence of DSR 
protocol. This convergence fact again leads to the betterment of Vegas in different mobile scenarios. In Figure 3, Packet delivery ratio is maintained by the DSR as mobility increases from the (pause time 10).

DSR have difficulties to maintain fresh routing information when nodes are moving fast. Practically all discarded packets were dropped because a stale routing table entry forwarded packets over a broken link. DSDV maintains only one route per destination; thus in case of broken link no alternative route can be found. DSDV seem to have generally better packet delivery ratio than DSR that reacts heavily to changes in mobility ratios.

At different pause times DSR performance can be considered on the average as compared to DSDV for average End-to-End Delay, the reason for this average delay is because Vegas has better estimation approach and also its capacity to detect any congestion in advance. Vegas attempts to keep the sending rate around a point estimated by the RTT samples. The idea is that if we increase the sending rate, and the RTT does not increase. However, if the RTT increases as we increase the sending rate, then we are not getting more bandwidth and instead are just taking up more space in the queues of intermediate than necessary [9] .DSDV has the highest end-to-end delay at pause time 10 due to high mobility and another reason is that DSDV protocol keeps packets in queues indefinitely until they are delivered to the next hop or the destination node. Therefore, it delivers the older packets rather than newer ones, and hence there is an increase in average end- to- end delay for DSDV protocol. As shown in graphs (Figure 5) at pause times 10 the DSDV was suffering from the highest end to end Delay as compared to DSR. DSR has somewhat better technique in this regard, since the destination replies only to the first arriving RREQ. This automatically favors the least congested route instead of the shortest route.

The reasons for packet dropping may be for example are that a packet is dropped due to congestion if the packet buffer at MAC layer is full when it arrives. When a collision is detected, CSMA does an exponential back off, which increases the delay for sending the packet. It makes the packet buffer to be full quickly. The packet drop ratio is high in DSDV at pause time 10 and gradually is decreasing and overall performance of both protocols in both TCP variants that are TCP Vegas and TCP Newreno DSR is performing well.

\section{CONCLUSION AND FUTURE WORK}

This paper is based on the compression of two well known protocols namely DSR and DSDV under two TCP variants known as TCP Vegas and TCP Newreno. From the simulation performed under three parameters i.e. Packet delivery ratio, Average End to End delay and total packets dropped, it can be concluded that DSDV has higher packet delivery ratio where as DSR in most cases. When we talk about the Average End to end delay as the above simulations are taken under TCP Vegas and TCP Newreno which emphasize the packet delay, rather than packet loss, so DSR has average performance, DSDV has highest end to end delay at pause time 10 due to high mobility. DSDV performs very poorly if we taken it from the perspective of total packets dropped at all pause times under both TCP variants.

The future work could be conducted with the analysis of MANET environment under different quality of service (QoS) issues such as node energy consumption, issues of hidden and exposed terminals, and constraints in mobility and traffic criteria. Also more MANET protocols like Hierarchal state routing protocol (HSR), temporarily ordered routing protocol (TORA), 
International Journal of Ad hoc, Sensor \& Ubiquitous Computing (IJASUC) Vol.4, No.4, August 2013

Preemptive Ad hoc on-demand distance-Vector routing protocol (PAODV). Dynamic MANET On-Demand (DYMO) can be added for better analysis and comparison of protocols under different TCP variants. Other TCP variants can also be tested. In future more parameters will be taken into consideration such as different traffic scenarios, congestion window size, number of connections etc.

\section{ACKNOWLEDGMENTS}

The authors wish to acknowledge Shams Laghari, Assistant Professor, Hamdard University, Karachi, Pakistan, for his continual support to carry out this work.

\section{REFERENCES}

[1] Param and Sharma, (2011), Performance study of Broadcast based Mobile Adhoc Routing Protocols AODV, DSR and DYMO. In International Journal of Security and its Applications, Vol.5, No. 1, pp 53-64.

[2] Juan, Josep and Miguel, (2005), Energy Efficiency of Load Balancing in MANET Routing Protocols. In Proceedings of the Sixth International Conference on Software Engineering, Artificial Intelligence, Networking and Parallel/Distributed Computing and First ACIS International Workshop on SelfAssembling Wireless Networks. pp. 476-483.

[3] Royer and Toh, (1999) A Review of Current Routing Protocols for Ad Hoc Mobile Wireless Networks, IEEE Personal Communications.

[4] Kurose and Ross, (2004) Computer Networking: A Top Down Approach Featuring the Internet, 3rd ed., Addison Wesley, $821 \mathrm{pp}$.

[5] Lu, Wang, Zhong and Bhargava, (2003) Study of Distance Vector Routing Protocols for Mobile Ad hoc Networks, Proceedings of the First IEEE international conference on Pervasive Computing and Communications (PerCom'03).

[6] Nehal and Roma, (2013), Performance Evaluation of AODV, DSDV and DSR Routing Protocols Using NS-2 Simulator. In International Journal of Engineering Research and Applications. Vol. 3, Issue 2, pp. 1825-1830.

[7] Johnson, Maltz, Hu, and Jetcheva, (2002). The Dynamic Source Routing Protocol for Mobile Ad Hoc Networks (DSR), Internet Engineering Task Force (IETF).

[8] Mukesh and Kumar, (2013). The Performance Evaluation of AODV and DSR (On-Demand-Driven) Routing Protocols using QuelNet 5.0 Simulator. In International Journal of Computer Science and Networks, Vol 2. Issue 2, pp. 29-34.

[9] Jong, Peter, Zhen and limin, (1995) Evaluation of TCP Vegas: Emulation and Experiment. Computer Science Department, University of Southern California, Los Angeles, CA. 